\title{
Mandenkan
}

MANDENIKAN Bulletin semestriel d'études linguistiques mandé

49 | 2013

Le maninka du Niokolo

\section{La prédication verbale et les marqueurs prédicatifs}

Section 4

\section{Denis Creissels}

\section{(2) OpenEdition}

Journals

Édition électronique

URL : https://journals.openedition.org/mandenkan/600

DOI : $10.4000 /$ mandenkan.600

ISSN : 2104-371X

Éditeur

Llacan UMR 8135 CNRS/Inalco

Édition imprimée

Date de publication : 1 juin 2013

Pagination : 38-44

ISSN : 0752-5443

Référence électronique

Denis Creissels, "La prédication verbale et les marqueurs prédicatifs », Mandenkan [En ligne], 49 | 2013, mis en ligne le 25 avril 2014, consulté le 16 janvier 2023. URL : http://journals.openedition.org/ mandenkan/600; DOI : https://doi.org/10.4000/mandenkan.600

Ce document a été généré automatiquement le 16 janvier 2023.

\section{(c) $)(1)(2)$}

Creative Commons - Attribution - Pas d'Utilisation Commerciale - Partage dans les Mêmes Conditions 4.0 International - CC BY-NC-SA 4.0

https://creativecommons.org/licenses/by-nc-sa/4.0/ 


\section{La prédication verbale et les marqueurs prédicatifs}

Section 4

Denis Creissels

\subsection{La construction prédicative verbale}

1 Comme c'est de manière générale le cas dans les langues de la famille mandé, la construction prédicative verbale en maninka du Niokolo se fait selon le schème $\mathrm{S}(\mathrm{O}) \mathrm{V}(\mathrm{X})$, où $\mathrm{S}, \mathrm{O}, \mathrm{V}$ et $\mathrm{X}$ sont mis pour sujet, objet, verbe et oblique respectivement.

2 En règle générale l'expression grammaticalisée du TAM et de la polarité (positif vs. négatif) se fait au moyen d'un marqueur prédicatif qui se place immédiatement après le sujet. On relève un seul cas dans lequel TAM et polarité ne sont pas encodés par un marqueur prédicatif succédant au sujet, mais par un suffixe verbal.

Dans la prédication verbale analytique, qui sera décrite en 9 , on a un schème superficiellement très semblable : $\mathrm{S}$ Aux $(\mathrm{O}) \mathrm{V}(\mathrm{X})$, avec un auxiliaire dont la position est comparable à celle d'un marqueur prédicatif mais qui s'en distingue par le fait que, ou bien il est fléchi lui-même comme un verbe, ou bien il ne manifeste pas de flexion mais peut par ailleurs assumer une fonction prédicative sans avoir à se combiner obligatoirement à un verbe.

\subsection{Les marqueurs prédicatifs}

4 Dans cette section, on s'intéresse uniquement aux marqueurs prédicatifs stricto sensu, mais il ne faut pas perdre de vue qu'à côté du système que forment ces marqueurs prédicatifs, l'expression de l'inaccompli met en jeu des constructions faisant appel à la copule locative en fonction d'auxiliaire (cf. 9.2, 9.3). 


\subsubsection{L'accompli-statif}

5 L'étiquette 'accompli-statif' est utilisée ici pour le couple de marqueurs prédicatifs ye (positif) / may (négatif) et pour le suffixe verbal - 'ta, qui exprime exactement la même valeur que ye et a aussi pour correspondant négatif may. La distribution de ye et - 'ta est la suivante :

- dans la construction transitive, c'est exclusivement ye qui s'utilise pour marquer l'accomplistatif ;

- dans la construction intransitive, avec la plupart des verbes c'est - 'ta qui est utilisé ;

- il existe toutefois une classe de verbes (désignés ici comme 'verbes qualificatifs') qui s'emploient en construction intransitive avec le prédicatif ye.

Le choix de l'étiquette 'accompli-statif' (plutôt que simplement 'accompli') tient au fait que comme c'est généralement le cas dans les parlers mandingues, en fonction de l'aspect lexical des verbes, la forme conventionnellement désignée comme accompli peut se référer à un processus dont le déroulement est antérieur à un certain repère ou à un état qui n'est pas nécessairement concevable comme résultant de la stabilisation d'un processus. Par exemple, l'accompli jaímfa-ta de jaímfa 's'éloigner' peut parfaitement s'utiliser, non seulement pour rendre ce que le français rendrait par s'être éloigné (référence à une distance résultant d'un processus d'éloignement) mais aussi comme équivalent de être loin en référence à deux entités occupant toutes les deux une position immuable dans l'espace, comme par exemple deux montagnes. L'analyse détaillée de ces interférences entre aspect lexical et polysémie du marqueur d'accompli-statif n'est toutefois pas abordée dans cette esquisse.

7 L'exemple (16) illustre l'emploi du couple ye / may avec une valeur d'accompli dans la construction transitive.

\begin{tabular}{|c|c|c|c|c|c|c|}
\hline (16) & a. & 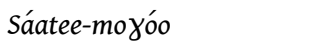 & $l u$ & yé & siláa & daídaa. \\
\hline & & village-personne.D & PL & ACPP & route.D & réparer \\
\hline & & \multicolumn{5}{|c|}{ ‘Les villageois ont réparé la route.’ } \\
\hline & b. & Watayáa & ye & siiisée & doo & múta. \\
\hline & & épervier.D & АСРP & poule.D & INDEF & attraper \\
\hline & & \multicolumn{5}{|c|}{ 'L'épervier a attrapé une poule.' } \\
\hline & c. & Yere-muta-láa & măy & yeze & múta. & \\
\hline & & poisson-attraper-NMAG.D & ACPN & poisson & attraper & \\
\hline & & \multicolumn{5}{|c|}{ 'Le pêcheur n’a pas attrapé de poisson.' } \\
\hline
\end{tabular}


8 L'exemple (17) illustre l'emploi du couple ye / may en construction transitive avec une valeur stative.

\begin{tabular}{|c|c|c|c|c|c|c|c|c|c|}
\hline \multirow[t]{3}{*}{ (17) } & a. & \multicolumn{2}{|l|}{ Á } & ye & \multicolumn{2}{|c|}{ mániyka-kán-o } & \multicolumn{3}{|l|}{ nóo. } \\
\hline & & \multicolumn{2}{|l|}{$3 \mathrm{SG}$} & АСРP & \multicolumn{2}{|c|}{ maninka-langue-D } & \multicolumn{3}{|c|}{ maîtriser } \\
\hline & & \multicolumn{8}{|c|}{ 'Il parle maninka.' } \\
\hline & & Á & \multicolumn{2}{|c|}{ běe } & ye & $a ́$ & dig-o & kánu & lée. \\
\hline & & \multicolumn{2}{|l|}{$3 \mathrm{SG}$} & tous & ACPP & $3 S G$ & enfant-D & -D & FOC \\
\hline & & \multicolumn{8}{|c|}{ 'Tous les gens aiment leurs enfants.' ${ }^{1}$} \\
\hline & c. & Á & $\operatorname{mag}$ & & abu-kár & & & ióo. & \\
\hline & & $3 S G$ & ACPP & & opéen-1 & sue-D & & naîtriser & \\
\hline & & ‘I n & arle & pas $\mathrm{f}$ & inçais.' & & & & \\
\hline
\end{tabular}

9 L'exemple (18) illustre l'emploi du couple - 'ta / may dans la construction intransitive.

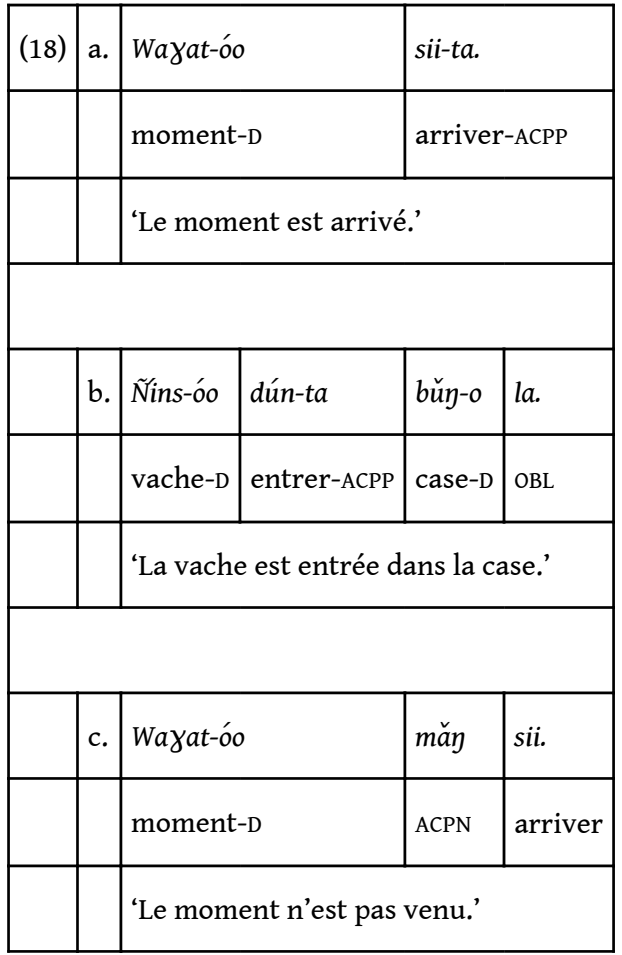


Enfin, l'exemple (19) illustre l'emploi du couple ye / may en construction intransitive, emploi qui implique toujours une valeur stative et qui est propre à une classe de verbes qualificatifs sur laquelle plus de détails seront donnés en 5.2.

\begin{tabular}{|c|c|c|c|c|c|c|c|c|}
\hline \multirow[t]{3}{*}{ (19) } & a. & Ñin & \multicolumn{2}{|l|}{ kónǒo } & \multicolumn{2}{|c|}{ dapatán-o } & ye & jay. \\
\hline & & DEM & \multicolumn{2}{|c|}{ oiseau.D } & \multicolumn{2}{|c|}{ aile-D } & ACPP & être_long \\
\hline & & \multicolumn{7}{|c|}{ 'Les ailes de cet oiseau sont longues.' } \\
\hline & b. & \multicolumn{2}{|l|}{ Bála-sub-óo } & \multicolumn{2}{|l|}{ ye } & \multicolumn{2}{|l|}{ dii } & lée. \\
\hline & & \multicolumn{2}{|c|}{ porc_épic-viande-D } & ACPF & & \multicolumn{2}{|c|}{ être_bon } & FOC \\
\hline & & \multicolumn{7}{|c|}{ ‘La viande du porc-épic est bonne.’ } \\
\hline & c. & \multicolumn{2}{|l|}{ Ñin } & \multicolumn{2}{|c|}{ múrǔ-y } & \multicolumn{2}{|l|}{ măy } & hii. \\
\hline & & \multicolumn{2}{|l|}{ DEM } & \multicolumn{2}{|c|}{ couteau-DEF } & \multicolumn{2}{|c|}{ ACPN } & être_bon \\
\hline & & ‘Ce couteau r & 'est $\mathrm{p}_{2}$ & s bon & & & & \\
\hline
\end{tabular}

11 Du point de vue morphologique, il faut noter l'existence de deux variantes du prédicatif $y e$, probablement apparentées étymologiquement entre elles mais dont le statut est différent: une variante $\eta e \sim \eta a$ dont l'emploi est obligatoire au contact de nasale, illustrée à l'ex. (20), et une variante ya qui peut s'employer en variation libre avec yeídans les autres contextes.

\begin{tabular}{|l|l|l|l|l|l|l|}
\hline$(20)$ & $\tilde{N}$ ì & baará- $\eta$ & $\eta e$ & $\eta$ & bátaa & lée. \\
\hline & DEM & travail-DEF & ACPP & 1SG & fatiguer & FOC \\
\hline \multicolumn{7}{|l}{ 'Ce travail m'a fatigué.' } \\
\hline
\end{tabular}

12 La variante $\gamma a$ du prédicatif d'accompli-statif positif est très peu employée en maninka du Niokolo. Elle correspond au prédicatif $x a$ qui marque l'accompli positif transitif dans d'autres variantes maninka du Sénégal Oriental.

\subsubsection{L'accompli récent}

Le maninka du Niokolo peut marquer l'accompli récent au moyen de deux prédicatifs synonymes, biriy et jaye $\sim$ jaya. Ces deux prédicatifs n'ont pas de correspondant négatif qui leur soit propre. 


\begin{tabular}{|l|l|l|l|l|l|}
\hline (21) & a. & A & birin & fúta & lée. \\
\hline & & 3SG & ACPR & arriver & FOC \\
\hline & & 'Il vient d'arriver.' & \\
\hline \multicolumn{5}{|c|}{} \\
\hline & b. & A & birí & tara & lée. \\
\hline & & 3SG & ACPR & partir & FOC \\
\hline & & 'Il vient de partir.' & \\
\hline &
\end{tabular}

\begin{tabular}{|c|c|c|c|c|c|c|c|c|}
\hline \multirow[t]{3}{*}{ (22) } & \multirow[t]{2}{*}{ a. } & $\eta$ & jayé & \multicolumn{2}{|c|}{ baar-óo } & \multicolumn{2}{|l|}{ kuraa } & lée. \\
\hline & & $1 S G$ & ACPR & \multicolumn{2}{|c|}{ travail-D } & \multicolumn{2}{|l|}{ finir } & FOC \\
\hline & & \multicolumn{7}{|c|}{ 'Je viens de finir le travail.' } \\
\hline & b. & I & jayé & mèn & fo, & tóññáa & lée & $m u$. \\
\hline & & $2 S G$ & ACPR & REL & dire & vérité.D & FOC & COPID \\
\hline & & & 10 & vien & de $d$ & re est vr & & \\
\hline
\end{tabular}

\subsubsection{Le potentiel}

14 L'étiquette 'potentiel' est appliquée ici au couple de prédicatifs se (positif) / mee (négatif), qui selon les contextes peuvent se rencontrer avec une valeur modale de potentiel ou avec une valeur plutôt aspectuelle d'habituel, parfois aussi avec une valeur de futur, la limite entre ces différentes valeurs étant souvent difficile à reconnaître au niveau des exemples concrets.

\begin{tabular}{|l|l|l|l|l|}
\hline$(23)$ & a. & Alá & mé. & log. \\
\hline & & Dieu & POTN & connaître \\
\hline & & \multicolumn{2}{|c|}{ 'On ne peut pas connaître Dieu.' } \\
\hline
\end{tabular}




\begin{tabular}{|c|c|c|c|c|c|c|c|c|c|}
\hline b. & Ñ̃oo & se & \multicolumn{3}{|l|}{ fii } & báyku-ñimmáa & lée & & to. \\
\hline & mil.D & РОТР & \multicolumn{2}{|c|}{ semer } & \multicolumn{2}{|c|}{ sol-bon.D } & FOC & & LOC \\
\hline & \multicolumn{9}{|c|}{ 'On sème le mil dans de la bonne terre.' } \\
\hline \multirow[t]{3}{*}{ c. } & Siisée & mee & túmbu & \multicolumn{2}{|l|}{ jay-óo } & $a ́$ & diz-o & lu & $m a$. \\
\hline & poule.D & POTN & ver & mauvais-D & donner & ler 3 3SG & \multicolumn{2}{|c|}{ enfant-D } & OBL \\
\hline & \multicolumn{9}{|c|}{ 'La poule ne donne pas un mauvais ver à ses poussins.' } \\
\hline d. & Find-óo & & \multicolumn{2}{|l|}{ se } & \multicolumn{3}{|l|}{ móo } & \multicolumn{2}{|l|}{ saañíi. } \\
\hline & \multicolumn{2}{|l|}{ fonio-D } & \multicolumn{2}{|l|}{ РОТР } & \multicolumn{2}{|c|}{ mûrir } & \multicolumn{3}{|c|}{ maintenant } \\
\hline & \multicolumn{9}{|c|}{ 'Le fonio devrait bientôt être mûr.' } \\
\hline e. & Bálǎa & & se & $a ́$ & la & \multicolumn{2}{|l|}{ tiy-óo } & fayi & lée. \\
\hline & \multicolumn{2}{|l|}{ porc_épic.D } & РОТР & $3 S G$ & GEN & poil-D & & lancer & FOC \\
\hline & 'Le porc-épic lar & $\mathrm{ce} / \mathrm{pe}$ & at lance & r ses piquants.' & & & & & \\
\hline f. & 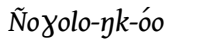 & lu & se & séněe & ke & dábǎa & & lé & la. \\
\hline & Niokolo-RESID-D & PL & РОТР & cultiver.D & faire & houe.D & & FOC & OBL \\
\hline & 'Les gens du Nio & colo cu & tivent & la houe.' & & & & & \\
\hline g. & $I$ & & se & 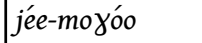 & & lu & & kóntoy. & \\
\hline & $2 S G$ & & POTP & là-personne.D & & PL & & saluer & \\
\hline & 'Tu salueras les & ens de & là-bas.' & & & & & & \\
\hline h. & $\tilde{N i \eta}$ & & & $e^{-x}$ & & & ke. & & \\
\hline & DEM & & & TTN & & & faire & & \\
\hline
\end{tabular}


'Ça ne se fait pas.'

L'expression non ambiguë de la potentialité s'obtient en combinant ces prédicatifs au verbe nóo 'pouvoir', comme en (24).

\begin{tabular}{|l|l|l|l|l|l|}
\hline$(24)$ & Ñin & mee & dólǒo & búla & nóo. \\
\hline & DEM & POTN & bière.D & laisser & pouvoir \\
\hline \multicolumn{6}{|l}{} \\
\multicolumn{4}{|l}{ 'Il n'arrive pas à abandonner la boisson.' }
\end{tabular}

\subsubsection{Le subjonctif et l'impératif}

\subsubsection{Le subjonctif}

16 L'étiquette 'subjonctif' est appliquée ici au couple de prédicatifs ye (positif) / kanáa (négatif). Le prédicatif ye du subjonctif positif est homonyme de ye 'accompli-statif positif, et présente la même variante $y e \sim$ ya au contact de nasale.

17 En phrase indépendante, le subjonctif exprime une valeur hortative (au positif) ou prohibitive (au négatif) - ex. (25). Pour son emploi dans la subordination, cf. section 22.

\begin{tabular}{|c|c|c|c|c|c|}
\hline$(25)$ & a. & $A$ & baabá & ye & náa! \\
\hline & & $3 \mathrm{SG}$ & père & SUBJP & venir \\
\hline & & \multicolumn{4}{|c|}{ ‘Que son père vienne !' } \\
\hline & b. & $\tilde{N} i \eta$ & suykutú- $\eta$ & ye & tara! \\
\hline & & DEM & fille-DEF & SUBJP & partir \\
\hline & & \multicolumn{4}{|c|}{ 'Que cette fille parte!' } \\
\hline & c. & Suykut-óo & kanáa & tałá & foloo! \\
\hline & & fille-D & SUBJN & partir & pour_l'instant \\
\hline & & \multicolumn{4}{|c|}{ 'Que la fille ne parte pas encore !' } \\
\hline
\end{tabular}

\subsubsection{L'impératif 2ème personne.}

Il existe une construction particulière d'impératif avec des sujets de 2ème personne. A l'impératif singulier (positif ou négatif), le sujet n'est pas exprimé. A l'impératif positif, 
on a un prédicatif zéro, alors que l'impératif négatif est marqué du même prédicatif kanáa que le subjonctif négatif, ce qui donne le paradigme illustré à l'ex. (26).

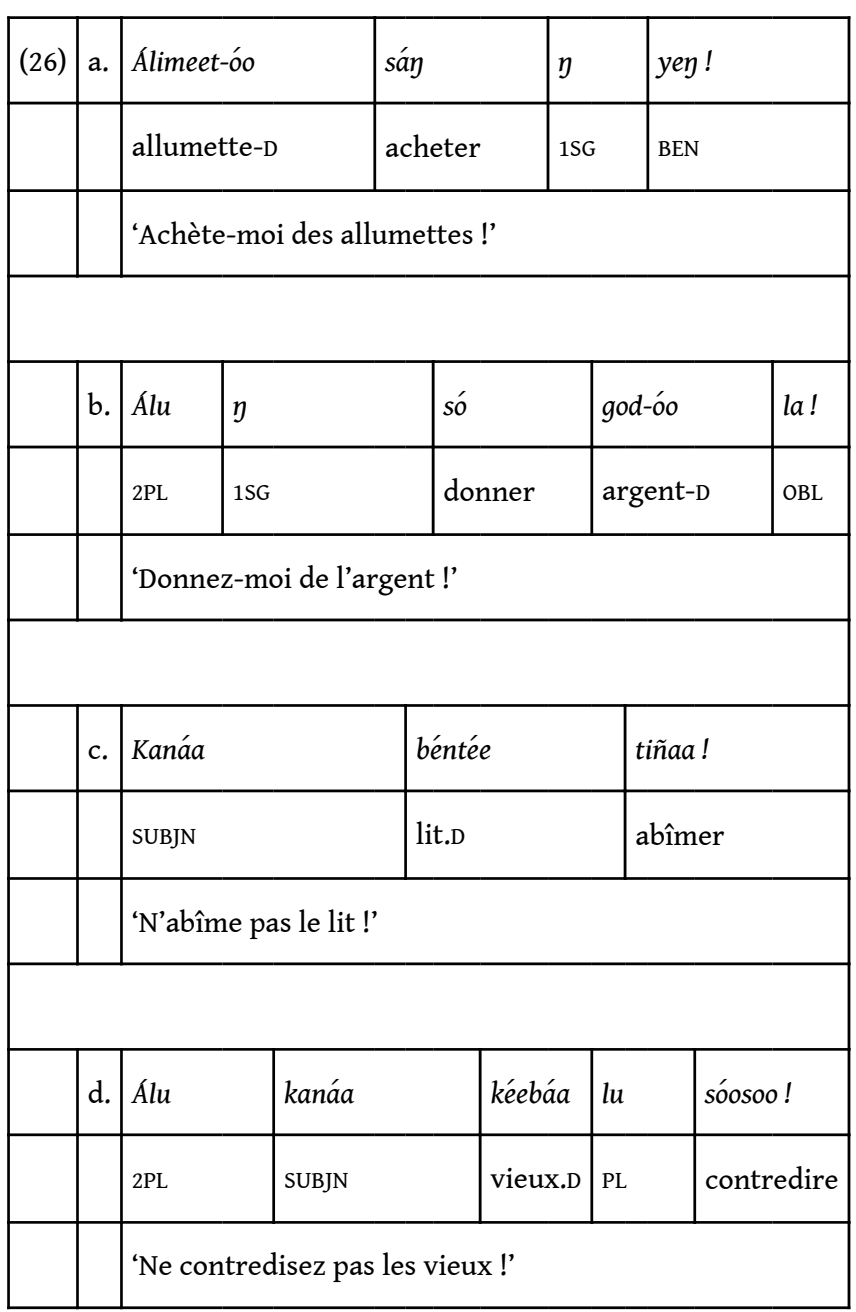

\subsubsection{L'impératif inclusif}

19 Le maninka du Niokolo a aussi une construction spéciale d'impératif inclusif (ou de première personne du pluriel). Dans cette construction, le prédicatif du subjonctif (positif ou négatif) est précédé d'un pronom inclusif aluín qui ne peut figurer dans aucun autre contexte - ex. (27).

\begin{tabular}{|c|c|c|c|c|c|c|}
\hline \multirow[t]{3}{*}{ (27) } & a. & \multicolumn{2}{|l|}{ Alúg } & \multicolumn{2}{|l|}{ ye } & taya! \\
\hline & & INCL & & SUBJP & F & partir \\
\hline & & \multicolumn{5}{|c|}{ ‘Partons !' } \\
\hline & b. & Alúg & ge & & gid-óo & núхü! \\
\hline
\end{tabular}




\begin{tabular}{|c|c|c|c|c|}
\hline & INCL & SUBJP & fusil-D & D cacher \\
\hline & \multicolumn{4}{|c|}{ ‘Cachons le fusil !' } \\
\hline \multirow[t]{2}{*}{ c. } & Alúy & \multicolumn{2}{|c|}{ kanaía } & tara! \\
\hline & \multicolumn{2}{|l|}{ INCL } & SUBJN & partir \\
\hline & \multicolumn{4}{|c|}{ 'Ne partons pas!' } \\
\hline d. & Alún & kana & dólo & mín! \\
\hline & INCL & SUBJN & bière & boire \\
\hline & ‘ $\mathrm{Ne} \mathrm{b}$ & uvons $\mathrm{p}$ & de bièr & \\
\hline
\end{tabular}

Ce pronom inclusif a manifestement pour origine la juxtaposition des pronoms de

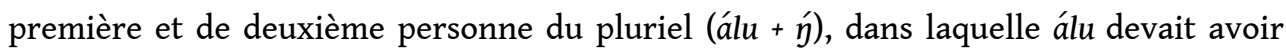
initialement une valeur d'interpellation. Toutefois la tonalité de alún n'est pas celle qu'on attendrait d'après cette décomposition, et c'est la raison pour laquelle il est analysé ici comme une unité à part (à la différence du mandinka où la même construction existe, mais avec une tonalité conforme à cette décomposition).

\subsubsection{L'obligatif}

21 Il existe enfin un prédicatif kámmu qui exprime l'obligation, et qui n'a pas de correspondant négatif - ex. (28). Avec ce prédicatif, le verbe est à la forme de l'infinitif en -la, ce qui suggère une grammaticalisation récente à partir d'un verbe auxiliaire, sur lequel je n'ai toutefois aucune hypothèse à proposer.

\begin{tabular}{|l|l|l|l|l|l|}
\hline (28) & I & kámmu & ñin & $k e$-la & lée. \\
\hline & 2SG & OBLIG & DEM & faire-INF & FOC \\
\hline \multicolumn{6}{|l}{ 'Tu dois faire cela.' } \\
\hline
\end{tabular}

\subsection{L'expression du passé}

Le marqueur de passé túy se place après le sujet. Si un prédicatif est aussi présent après le sujet, túg précède le prédicatif. A l'accompli en - 'ta, túy se place entre le sujet et le verbe. 


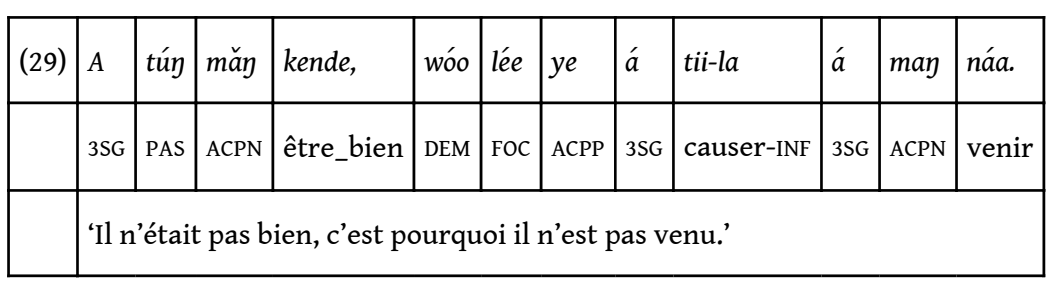

23 L'adverbe nuy 'autrefois', qui en mandinka s'est grammaticalisé comme marqueur de passé, est aussi attesté en maninka du Niokolo, mais rien n'indique qu'il soit en voie de grammaticalisation, et l'expression grammaticalisée du passé se fait en maninka du Niokolo avec le même marqueur tuín que dans la plupart des variétés mandingues.

\section{NOTES}

1. Sur l'emploi de á bee' au sens de 'tout le monde', cf. section 10.4.

\section{AUTEUR}

\section{DENIS CREISSELS}

Université de Lyon

Denis.Creissels@univ-lyon2.fr 